\title{
Correction to: Fun with Functors
}

\section{Anto Aravinth and Srikanth Machiraju}

\section{Correction to:}

Chapter 8 in: A. Aravinth and S. Machiraju, Beginning Functional JavaScript: Uncover the Concepts of Functional Programming with EcmaScript 8

https://doi.org/10.1007/978-1-4842-4087-8_8

The original version of the chapter was inadvertently published with an incorrect code in chapter 8 and that has been corrected now.

The updated version of the chapter can be found at https://doi.org/10.1007/978-1-4842-4087-8_8 